\title{
Internal resonance and nonlinear response of an axially moving beam: two numerical techniques
}

\author{
Mergen H. Ghayesh* and Marco Amabili \\ Department of Mechanical Engineering, McGill University, Montreal, Quebec, Canada H3A 0C3
}

(Received June 21, 2012, Revised September 2, 2012, Accepted September 8, 2012)

\begin{abstract}
The nonlinear resonant response of an axially moving beam is investigated in this paper via two different numerical techniques: the pseudo-arclength continuation technique and direct time integration. In particular, the response is examined for the system in the neighborhood of a three-to-one internal resonance between the first two modes as well as for the case where it is not. The equation of motion is reduced into a set of nonlinear ordinary differential equation via the Galerkin technique. This set is solved using the pseudo-arclength continuation technique and the results are confirmed through use of direct time integration. Vibration characteristics of the system are presented in the form of frequency-response curves, time histories, phase-plane diagrams, and fast Fourier transforms (FFTs).
\end{abstract}

Keywords: axially moving beams; vibrations; stability; bifurcation; internal resonance

\section{Introduction}

The demand for structural elements such as beams(Ghayesh 2011, Ghayesh et al. 2011, Ghayesh et al. 2011, Darabi et al. 2012, Ghayesh 2012, Ghayesh et al. 2012, Ghayesh et al. 2012), plates (Amabili et al. 2006, Amabili 2010, Amabili and Carra 2012) and shells is continuously increasing, largely due to their growing application in industry. Among these structural elements, axially moving systems arise in a large class of mechanical, industrial, and automotive applications. Textile fibers, paper sheets, band saw blades, robot arms, conveyor belts, and magnetic tapes are just a few examples. These widespread applications motivated a substantial amount of research in this area.

As reviewed by Wickert and Mote (Wickert and Mote 1988) and later by Chen (2005), dynamics of axially moving systems has been studied extensively in literature. One such example is (Naguleswaran and Williams 1968, Shih 1971, Simpson 1973, Holmes 1978, Thurman and Mote 1969). However, a thorough literature review on this topic will not be undertaken in this paper (interested readers are referred to Wickert and Mote (1988) and Chen (2005).

These early studies were pursued and extended by: Chen and co-workers (Tang et al. 2009, Chen and Chen 2010, Chen and Ding 2010, Huang et al. 2011, Yang et al. 2012), who considered string and different beam models of the system and employed different analytical and numerical methods; Marynowski and co-workers (Marynowski and Kapitaniak 2002, Marynowski and Kapitaniak 2007),

\footnotetext{
* Corresponding author, Dr., E-mail: mergen.hajghayesh@mail.mcgill.ca
} 
who considered several energy dissipation mechanisms in the model; Pellicano and Vestroni (Pellicano and Vestroni 2002), who investigated the dynamics of high-speed axially moving systems; Suweken and Van Horssen (Suweken and Van Horssen 2003), who investigated the vibrations of the system with weak nonlinearity and found several internal resonances in the system dynamics; Huang et al. (2011), who employed the method of harmonic balance to investigate the system dynamics; Pakdemirli and co-workers (Pakdemirli et al. 1994, Pakdemirli and Ulsoy 1997, Pakdemirli and Özkaya 1998, Öz et al. 2001, Pakdemirli and BoyacI 2003, Burak Özhan and Pakdemirli 2010), who conducted systematic research in this area by employing some perturbation techniques; Stylianou and Tabarrok (Stylianou and Tabarrok 1994), who used the finite element method to examine the system dynamics; and Nguyen and Hong (2011), who developed a novel control algorithm to suppress the transverse vibrations of an axially moving web.

Recently, further investigations on this topic have been conducted by the first author and coworkers (Ghayesh and Khadem 2007, Ghayesh 2008, Ghayesh 2009, Ghayesh 2010, Ghayesh and Balar 2010, Ghayesh et al. 2010, Ghayesh 2011a, Ghayesh 2011b, Ghayesh and Moradian 2011, Ghayesh 2012a, Ghayesh 2012b, Ghayesh 2012c, Ghayesh and Amabili 2012, Ghayesh et al. 2012, Ghayesh et al. 2012, Ghayesh et al. 2012, Ghayesh et al. 2012, Kazemirad et al. 2012). These analyses involved various system models such as strings and Euler-Bernoulli, Rayleigh, and Timoshenko beam models. The effect of a partial foundation on the system dynamics was investigated in (Ghayesh 2008, Ghayesh 2009). The dynamics of the system made of laminated composite materials were investigated in (Ghayesh et al. 2010).

In the present study, the sub-critical resonant response of an axially moving beam possessing a three-to-one internal resonance between the first two modes is obtained using the pseudo-arclength continuation technique and direct time integration; a higher order discretization is conducted so as to study modal interactions and internal resonances as well as to obtain fairly accurate results. The analysis also includes the case without internal resonances between the first two modes. The results are presented in the form of frequency-response curves, time histories, phase-plane portraits, and fast Fourier transforms (FFTs). It is shown that the results obtained from the above two methods are in excellent agreement.

\section{Equation of motion and methods of solution}

As shown in Fig. 1, consider a simply supported axially moving beam of length $L$, constant density $\rho$, cross-sectional area $A$ and Young's modulus $E$ which is traveling at a constant axial speed $V$. There are two forces to be considered, namely $P$ and $\hat{F}(\hat{x}, \tau)=\hat{F}(\hat{x}) \cos (\omega \tau)$; the former being a pretension and the latter a harmonic transverse force distributed along the entire length of the beam. Furthermore, $\hat{x}$ denotes a Lagrangian coordinate.

The dimensionless form of the equation of motion of this system has been given previously in (Huang et al. 2011) as follows

$$
\frac{\partial^{2} w}{\partial t^{2}}+2 v \frac{\partial^{2} w}{\partial x \partial t}+\left(v^{2}-1\right) \frac{\partial^{2} w}{\partial x^{2}}+v_{f}^{2} \frac{\partial^{4} w}{\partial x^{4}}=\frac{3}{2} v_{1}^{2}\left(\frac{\partial w}{\partial x}\right)^{2} \frac{\partial^{2} w}{\partial x^{2}}+F(x) \cos (\Omega t)
$$

in which 


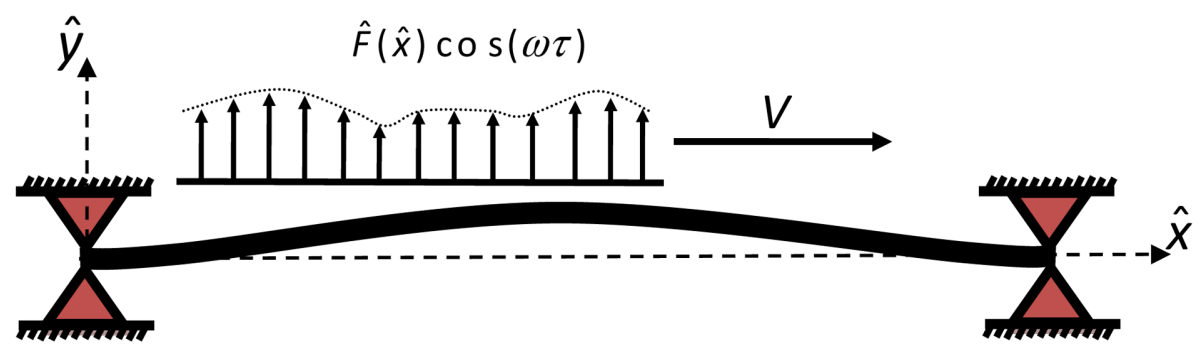

Fig. 1 Schematic representation of an axially moving beam subjected to a transverse distributed harmonic excitation force

$$
\begin{gathered}
x=\frac{\hat{x}}{L}, w=\frac{\hat{w}}{L}, t=\tau \sqrt{\frac{P}{\rho A L^{2}}}, v=V \sqrt{\frac{\rho A}{P}} \\
v_{1}=\sqrt{\frac{E A}{P}}, v_{f}=\sqrt{\frac{E I}{P L^{2}}}, F=\frac{\hat{F} L}{P}, \Omega=\omega \sqrt{\frac{\rho A L^{2}}{P}}
\end{gathered}
$$

where $x$ denotes the dimensionless counterpart of $\hat{x} ; w$ and $\hat{w}$ represent the dimensionless and dimensional transverse displacements; $\tau$ and $t$ represent the dimensional and dimensionless time; $F$ denotes the dimensionless forcing amplitude; and $\Omega$ is the dimensionless excitation frequency. Eq. (1) has been derived under the following assumptions: (i) rotatory inertia and shear deformations are neglected; (ii) only the transverse displacement is considered (Marynowski and Kapitaniak 2002, Ghayesh 2010, Huang et al. 2011); (iii) the type of nonlinearity is geometric ; (iv) the beam has a uniform cross-section; (v) the relation between the curvature and the displacement is assumed to be linear ; (vi) the nonlinearity is due to the stretching effect of the mid-plane of the beam; (vii) the equation is truncated at third order.

Exciting only the first mode by $f_{1} \phi_{1}(x)$, where $\phi_{1}(x)$ is the first eigenfunction for the transverse vibrations of a hinged-hinged stationary beam, as well as employing the well-known Galerkin method for Eq. (1) results in

$$
\begin{gathered}
\sum_{j=1}^{N}\left(\int_{0}^{1} \phi_{i} \phi_{j} d x\right) \ddot{q}_{j}+2 v \sum_{j=1}^{N}\left(\int_{0}^{1} \phi_{i} \phi_{j}^{\prime} d x\right) \dot{q}_{j}+\left(v^{2}-1\right) \sum_{j=1}^{N}\left(\int_{0}^{1} \phi_{i} \phi_{j}^{\prime \prime} d x\right) q_{j} \\
+v_{f}^{2}\left(\sum_{j=1}^{N} \int_{0}^{1} \phi_{i} \phi_{j}^{\prime \prime \prime} d x\right) q_{j}=\frac{3}{2} v_{1}^{2}\left(\sum_{j=1}^{N} \sum_{k=1}^{N} \sum_{l=1}^{N} \phi_{i} \phi_{j}^{\prime \prime} \phi_{k}^{\prime} \phi_{l}^{\prime} d x\right) q_{j} q_{k} q_{l} \\
+\int_{0}^{1} \phi_{i} f_{1} \phi_{1} d x \cos \Omega t, \text { for } i=1,2,3, \ldots, N
\end{gathered}
$$

where $\phi_{i}(x)$ is the ith eigenfunction of a hinged-hinged beam; dot and prime denote the differentiation with respect to dimensionless time and axial coordinate, respectively. Eq. (3) forms a set of second order nonlinear ordinary differential equations; however, most numerical techniques can best handle first order ordinary differential equations. Therefore, Eq. (3) is transformed into a set of first order nonlinear ordinary differential equations via $y_{i}=\dot{q}_{i}$ for $i=1,2, \cdots, N$, yielding $2 N$ first order nonlinear ordinary differential equations. In this paper, twelve $(N=6)$ first order nonlinear ordinary differential equations with coupled terms are solved using the AUTO code (Doedel et al. 1998), which uses the pseudo-arclength continuation method. Direct time integration 
is conducted using a variable step-size Runge-Kutta method to validate the results obtained via the pseudo-arclength continuation technique. It should also be noted that although there is no damping in the equation of motion, the numerical simulations include a simple viscous damping with the dimensionless coefficient of $\mu$.

\section{Resonant response of the system possessing a three-to-one internal resonance between the first two modes}

In this section, the resonant response of the system with a three-to-one internal resonance is examined. Two cases with different damping coefficients are considered, and it is shown that the damping of the system may change the system dynamics substantially. The frequency-response curves of the system are first plotted using the pseudo-arclength continuation technique, and then several points on those curves are tested via direct time integration.

The following parameters have been selected in the analysis of the first case: $v=0.6, v_{f}=0.173$, $v_{1}=33.526, \mu=0.07, f_{1}=0.0055$. The system with the first two numerical values given above undergoes a three-to-one internal resonance between the first two modes. Solving the linear part of Eq. (3) gives the following linear natural frequencies for the first two modes: $\omega_{1}=2.8172$ and $\omega_{2}=8.4984\left(\omega_{2} \approx 3 \omega_{1}\right)$ confirms that a three-to-one internal resonance occurs. The (maximum) amplitude of oscillation of this system in the neighborhood of the fundamental natural frequency is shown in Fig. 2; $q_{1}$ and $q_{2}$ are the first and second generalized coordinates. The curves have been obtained using the pseudo-arclength continuation technique and the symbols (with white filling) have been obtained by direct time integration. It is clear that the two are in excellent agreement. Theoretically, as the excitation frequency is increased, the bent section of the curve corresponding to the first generalized coordinate is to the right, showing hardening-type nonlinearity. As seen in Fig. 2(a), theoretically, as the excitation frequency is increased from $\Omega=0.6 \omega_{1}$, the $q_{1}$ amplitude increases accordingly until point $A\left(\Omega=1.0648 \omega_{1}\right)$, where stability is lost via a limit point bifurcation. The amplitude of this unstable solution decreases until point $B\left(\Omega=1.0620 \omega_{1}\right)$, where stability is
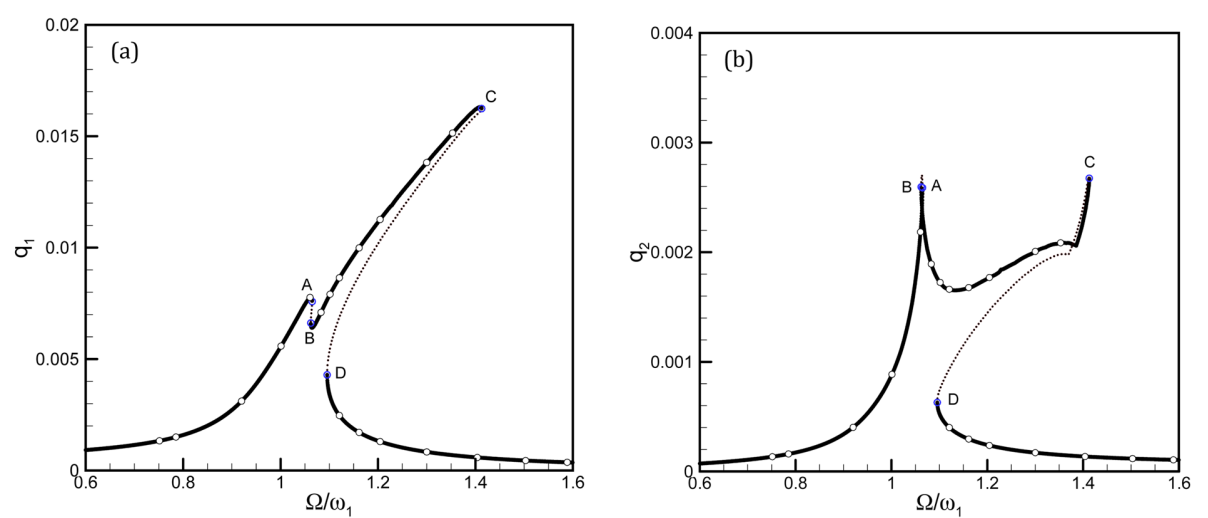

Fig. 2 The frequency-response curve of the system with $\mu=0.07$ possessing a three-to-one internal resonance between the first two modes, i.e., $\omega_{2} \approx 3 \omega_{1}$ : (a) the amplitude of the first generalized coordinate and (b) the amplitude of the second generalized coordinate. Bold and dotted lines represent the stable and unstable solutions, respectively. Symbols with white filling show the results obtained from direct time integration, and the blue symbols show the bifurcation points 
regained; the energy is transferred from the first generalized coordinate to the second one. Increasing the excitation frequency causes the $q_{1}$ amplitude to increase until point $C\left(\Omega=1.4127 \omega_{1}\right)$ is hit, where the motion becomes unstable once again by means of a limit point bifurcation. As the excitation frequency is decreased, this now unstable solution regains its stability at point $D(\Omega=$

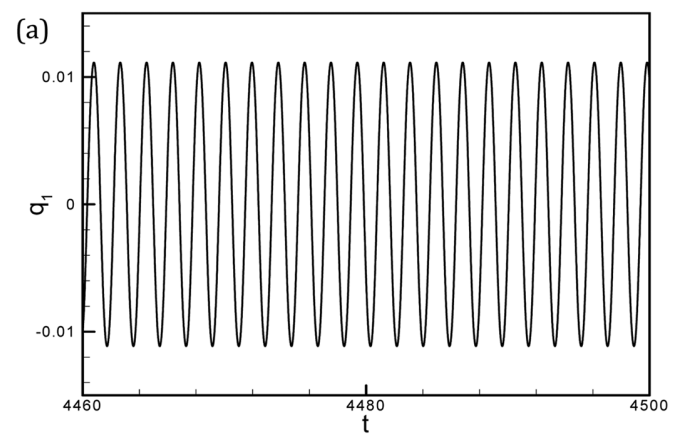

(b)

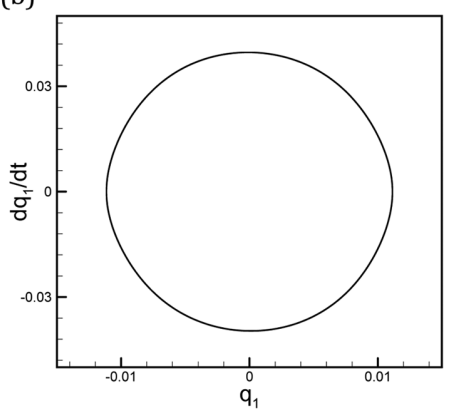

(c)

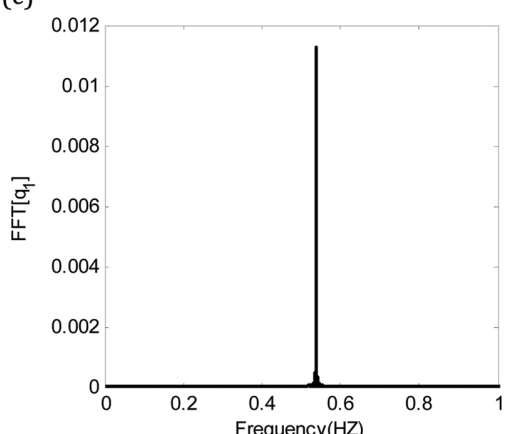

Fig. 3 Periodic oscillations of the system of Fig. 2 at $\Omega=1.2 \omega_{1}$ : (a) the time history, (b) phase-plane diagrams, and (c) FFT of the $q_{1}$ motion
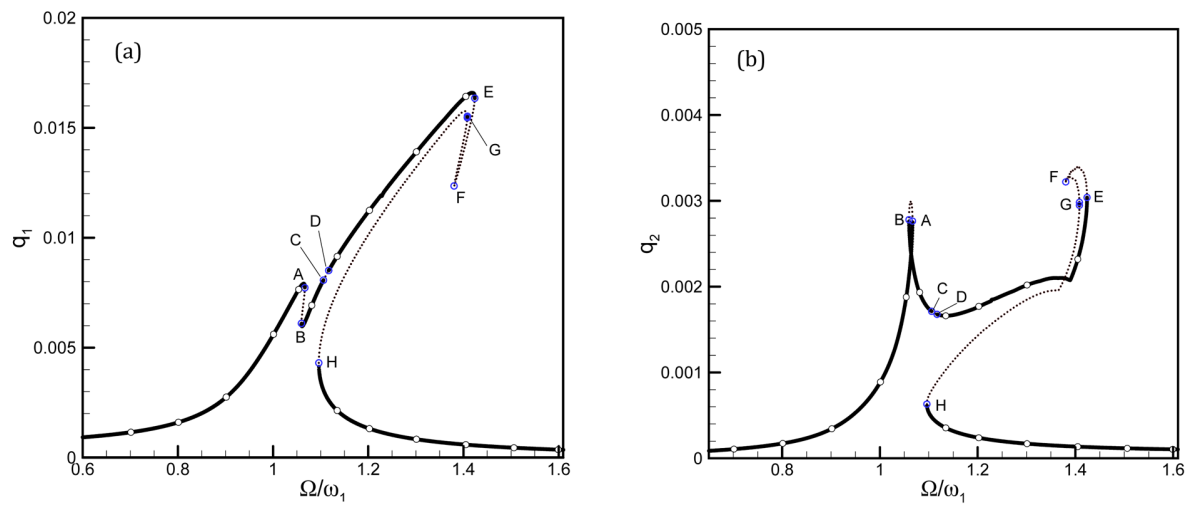

Fig. 4 The frequency-response curve of the system with $\mu=0.045$ possessing a three-to-one internal resonance between the first two modes, i.e., $\omega_{2} \approx 3 \omega_{1}$ : (a) the amplitude of the first generalized coordinate and (b) the amplitude of the second generalized coordinate. Bold and dotted lines represent the stable and unstable solutions, respectively. Symbols with white filling show the results obtained from direct time integration, and the blue symbols show the bifurcation points 
$1.0956 \omega_{1}$ ) and maintains it until $\Omega=1.6 \omega_{1}$. Typical features of a periodic motion at $\Omega=1.2 \omega_{1}$ are shown in Fig. 3. It is also seen in Fig. 2 that the only type of bifurcation is limit point; there is no torus bifurcation.

The second case to be considered is similar to the first, having almost all of the same parameters, except a lower damping coefficient (i.e., $\mu=0.045$ ). The frequency response of this case is shown in Fig. 4. As seen in this figure, hardening nonlinearity is present, and both the numerical results are in agreement. As seen in Fig. 4(a), theoretically, increasing the excitation frequency causes the $q_{1}$ amplitude, which is stable, to increase accordingly until the first limit point at point $A\left(\Omega=1.0667 \omega_{1}\right)$ is reached. By slightly decreasing the excitation frequency, the response becomes unstable and lasts until the second limit point at $B\left(\Omega=1.0595 \omega_{1}\right)$, where the stability is regained. As the excitation

(a)

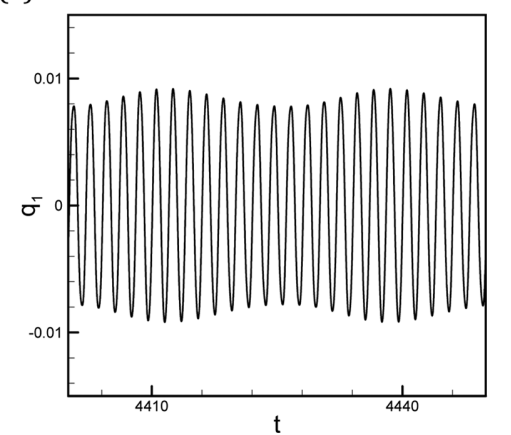

(b)

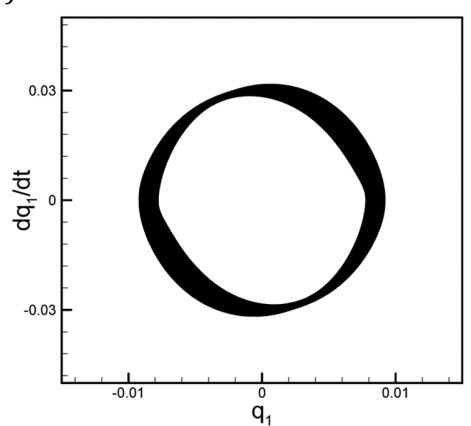

(c)

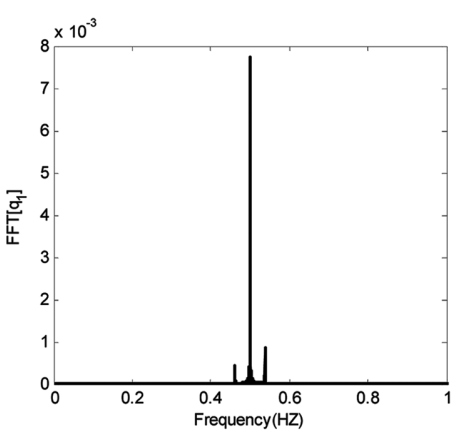

(d)

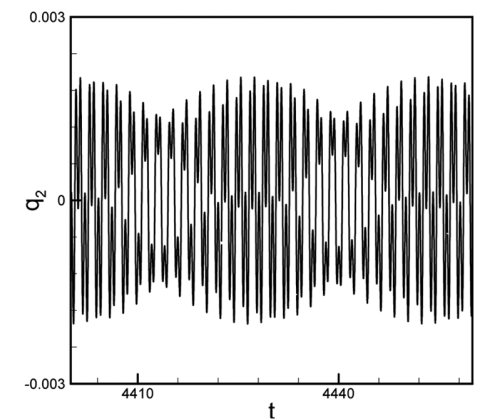

(e)

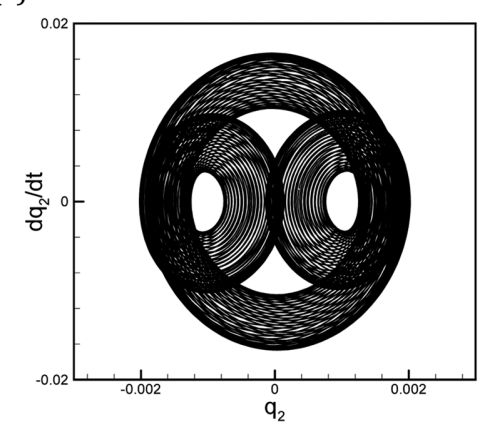

(f)

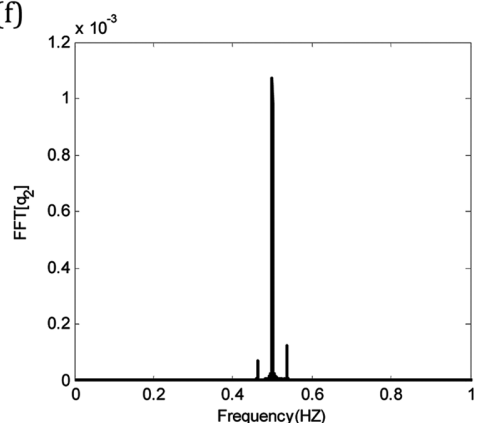

Fig. 5 Quasiperiodic oscillations of the system of Fig. 4 at $\Omega=1.115031 \omega_{1}$ : (a) the time history, (b) phaseplane diagram and (c) FFT of the $q_{1}$ motion; (d-f) the same characteristics of (a-c) of the $q_{2}$ motion 
frequency is increased further, the stability of the periodic response is lost at point $C\left(\Omega=1.1058 \omega_{1}\right)$, via a torus bifurcation. The stability is regained at point $D\left(\Omega=1.1170 \omega_{1}\right)$ via the second torus bifurcation. In the frequency range between points $C$ and $D$, the periodic solution is unstable, implying that there might be another type of motion present, such as quasiperiodic or chaotic. Figs. 5(a)-(c) shows (a) the time history of the $q_{1}$ motion, (b) the phase-plane portrait for the $q_{1}$ motion, (c) the FFT of the $q_{1}$ motion at $\Omega=1.115031 \omega_{1}$ - Figs. 5(d)-(f) shows the same characteristics of Figs. 5(a)-(c) for the $q_{2}$ motion. As seen in this figure, the amplitudes are modulated and the response is quasiperiodic. As the excitation frequency is increased further (Fig. 4(a)), the stability is lost once again at point $E\left(\Omega=1.4238 \omega_{1}\right)$ via a limit point bifurcation. There is another limit point bifurcation at point $F\left(\Omega=1.3802 \omega_{1}\right)$. In the vicinity of point $G\left(\Omega \approx 1.4082 \omega_{1}\right)$ the stability is initially regained but lost very soon afterward. This unstable solution lasts until point $H\left(\Omega=1.0961 \omega_{1}\right)$ is reached, where the motion becomes stable via a limit point bifurcation. It is important to note that, as opposed to the previous case (Fig. 2), torus bifurcations and quasiperiodic oscillations are observed in the dynamical behavior of the system with $\mu=0.045$ (see Figs. 4 and 5; compare Figs. 2 and 4).

\section{Resonant response of the system with no internal resonances between the first two modes}

The frequency-response curve of the system for the first generalized coordinate, with the following dimensionless parameters $v=0.2, v_{f}=0.173, v_{1}=33.526, \mu=0.06, f_{1}=0.0055$ is shown in Fig. 6. With these parameters, the first linear natural frequency is determined as: $\omega_{1}=3.4932$. As shown in this figure, the nonlinearity type is hardening.; it was found that, theoretically, as the excitation frequency is increased from $\Omega=0.6 \omega_{1}$, the $q_{1}$ amplitude increases accordingly until the first limit point at point $A\left(\Omega=1.3673 \omega_{1}\right)$ is hit, where it loses stability. The stability is regained via the

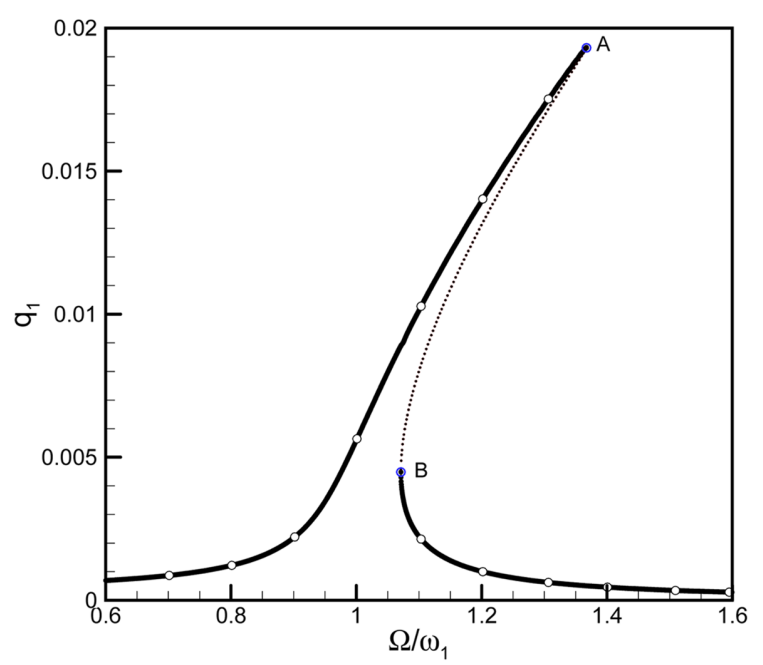

Fig. 6 The frequency-response curve of the system without any internal resonances between the first two modes. Bold and dotted lines represent the stable and unstable solutions, respectively. Symbols with white filling show the results obtained from direct time integration, and the blue symbols show the bifurcation points 
(a)

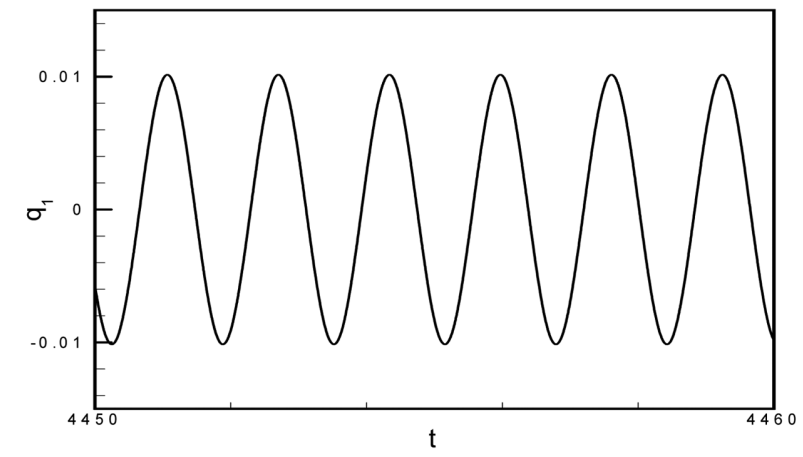

(b)

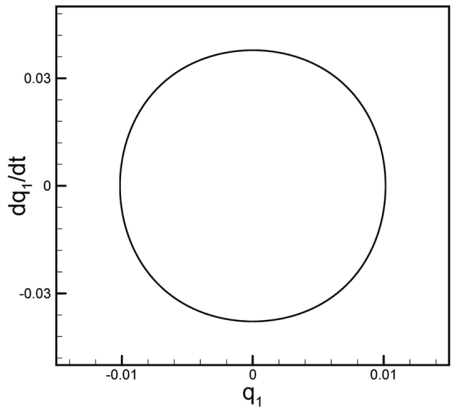

(C)

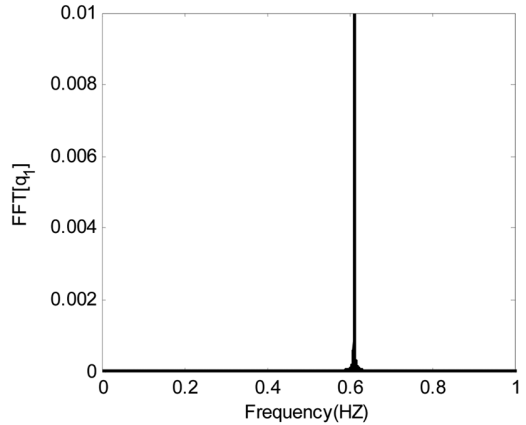

Fig. 7 Periodic oscillations of the system of Fig. 6 at $\Omega=1.1 \omega_{1}$ : (a) the time history, (b) phase-plane diagram, and (c) FFT of the $q_{1}$ motion

second limit point bifurcation at point $B$, where $\Omega=1.0711 \omega_{1}$. This now stable solution lasts until $\Omega=1.6 \omega_{1}$. Since the system is away from internal resonances between the first two modes, there is no energy transferred from the first mode to the second. Limit point bifurcation is the only type of bifurcation that is observed in the dynamical behavior of the system. Typical vibration characteristic of the system for $\Omega=1.1 \omega_{1}$ are shown in Fig. 7 .

\section{Conclusions}

The transverse, nonlinear resonant response of an axially moving beam possessing a three-to-one internal resonance between the first two modes as well as the case without any internal resonance between the first two modes have been investigated numerically. The pseudo-arclength continuation technique was used to plot the frequency-response curves. The stable solution branches were validated using direct time integration. The vibration characteristics of the system in the range, beyond the first torus bifurcation and before the second one were examined and it was shown that the system displays a quasiperiodic motion. The typical characteristics of this quasiperiodic motion were investigated via time histories, phase-plane plots, and FFTs. It was shown that the results obtained from both numerical techniques are in excellent agreement. This study contributed to the current knowledge on this topic by detecting new phenomena such as the occurrence of torus bifurcations in the system dynamics, which are hard to detect analytically. 


\section{References}

Amabili, M. (2010), "Geometrically nonlinear vibrations of rectangular plates carrying a concentrated mass", $J$. Sound Vib., 329(21), 4501-4514.

Amabili, M. and Carra, S. (2012), "Experiments and simulations for large-amplitude vibrations of rectangular plates carrying concentrated masses", J. Sound Vib., 331(1), 155-166.

Amabili, M., Pellegrini, M., Righi, F. and Vinci, F. (2006), "Effect of concentrated masses with rotary inertia on vibrations of rectangular plates", J. Sound Vib., 295(1-2), 1-12.

Burak Özhan, B. and Pakdemirli, M. (2010), "A general solution procedure for the forced vibrations of a system with cubic nonlinearities: Three-to-one internal resonances with external excitation", J. Sound Vib., 329(13), 2603-2615.

Chen, L.Q. (2005), "Analysis and control of transverse vibrations of axially moving strings", Appl. Mech. Rev., 58(2), 91-116.

Chen, L.Q. and Chen, H. (2010), "Asymptotic analysis on nonlinear vibration of axially accelerating viscoelastic strings with the standard linear solid model", J. Eng. Math., 67(3), 205-218.

Chen, L.Q. and Ding, H. (2010), "Steady-State transverse response in coupled planar vibration of axially moving viscoelastic beams", J. Vib. Acoust., 132(1), 011009.

Darabi, M.A., Kazemirad, S. and Ghayesh, M.H. (2012), "Free vibrations of beam-mass-spring systems: analytical analysis with numerical confirmation", Acta Mech. Sinica, 28 (2), 468-481.

Doedel, E.J., Champneys, A.R., Fairgrieve, T.F., Kuznetsov, Y.A., Sandstede, B. and Wang, X. (1998), AUTO 97: Continuation and Bifurcation Software for Ordinary Differential Equations (with HomCont), Concordia University, Montreal, Canada.

Ghayesh, M.H. (2011), "On the natural frequencies, complex mode functions, and critical speeds of axially traveling laminated beams: Parametric study", Acta Mech. Solida Sin., 24(4), 373-382.

Ghayesh, M.H. (2008), "Nonlinear transversal vibration and stability of an axially moving viscoelastic string supported by a partial viscoelastic guide", J. Sound Vib., 314(3-5), 757-774.

Ghayesh, M.H. (2009), "Stability characteristics of an axially accelerating string supported by an elastic foundation", Mech. Mach. Theory, 44(10), 1964-1979.

Ghayesh, M.H. (2010), "Parametric vibrations and stability of an axially accelerating string guided by a nonlinear elastic foundation", Int. J. Nonlinear Mech., 45(4), 382-394.

Ghayesh, M.H. (2011a), "Nonlinear forced dynamics of an axially moving viscoelastic beam with an internal resonance", Int. J. Mech. Sci., 53(11), 1022-1037.

Ghayesh, M.H. (2011b), "On the natural frequencies, complex mode functions, and critical speeds of axially traveling laminated beams: parametric study”, Acta Mech. Solida Sin., 24(4), 373-382.

Ghayesh, M.H. (2011c), "Parametrically excited viscoelastic beam-spring systems: Nonlinear dynamics and stability", Struct. Eng. Mech., 40(5), 705-718.

Ghayesh, M.H. (2012a), "Coupled longitudinal-transverse dynamics of an axially accelerating beam", J. Sound Vib., 331(23), 5107-5124.

Ghayesh, M.H. (2012b), "Nonlinear dynamic response of a simply-supported Kelvin-Voigt viscoelastic beam, additionally supported by a nonlinear spring", Nonlinear Anal. Real., 13(3), 1319-1333.

Ghayesh, M.H. (2012c), "Stability and bifurcations of an axially moving beam with an intermediate spring support", Nonlinear Dynam., 69(1-2), 193-210.

Ghayesh, M.H. (2012d), "Subharmonic dynamics of an axially accelerating beam", Arch. Appl. Mech., in press.

Ghayesh, M.H., Alijani, F. and Darabi, M.A. (2011), "An analytical solution for nonlinear dynamics of a viscoelastic beam-heavy mass system", J. Mech. Sci. Technol., 25(8), 1915-1923.

Ghayesh, M.H. and Amabili, M. (2012), "Steady-state transverse response of an axially moving beam with time dependent axial speed", Int. J. Nonlinear Mech., (in press).

Ghayesh, M.H., Amabili, M. and Païdoussis, M.P. (2012), "Nonlinear vibrations and stability of an axially moving beam with an intermediate spring-support: two-dimensional analysis", Nonlinear Dynam., in press.

Ghayesh, M.H. and Balar, S. (2010), "Non-linear parametric vibration and stability analysis for two dynamic models of axially moving Timoshenko beams", Appl. Math. Model., 34(10), 2850-2859.

Ghayesh, M.H., Kafiabad, H.A. and Reid, T. (2012), "Sub- and super-critical nonlinear dynamics of a 
harmonically excited axially moving beam", Int. J. Solids Struct., 49(1), 227-243.

Ghayesh, M.H., Kazemirad, S. and Amabili, M. (2012), "Coupled longitudinal-transverse dynamics of an axially moving beam with an internal resonance", Mech. Mach. Theory, 52, 18-34.

Ghayesh, M.H., Kazemirad, S. and Darabi, M.A. (2011), "A general solution procedure for vibrations of systems with cubic nonlinearities and nonlinear/time-dependent internal boundary conditions", J. Sound Vib., 330(22), $5382-5400$.

Ghayesh, M.H., Kazemirad, S., Darabi, M.A. and Woo, P. (2012), "Thermo-mechanical nonlinear vibration analysis of a spring-mass-beam system", Arch. Appl. Mech., 82(3), 317-331.

Ghayesh, M.H., Kazemirad, S. and Reid, T. (2012), "Nonlinear vibrations and stability of parametrically exited systems with cubic nonlinearities and internal boundary conditions: A general solution procedure", Appl. Math. Model., 36, 3299-3311.

Ghayesh, M.H. and Khadem, S.E. (2007), "Non-linear vibration and stability analysis of a partially supported conveyor belt by a distributed viscoelastic foundation", Struct. Eng. Mech., 27(1), 17-32.

Ghayesh, M.H. and Moradian, N. (2011), "Nonlinear dynamic response of axially moving, stretched viscoelastic strings", Arch. Appl. Mech., 81(6), 781-799.

Ghayesh, M.H., Païdoussis, M.P. and Amabili, M. (2012), "Subcritical parametric response of an axially accelerating beam", Thin Wall. Struct., 60, 185-193.

Ghayesh, M.H., Yourdkhani, M., Balar, S. and Reid, T. (2010), "Vibrations and stability of axially traveling laminated beams", Appl. Math. Comput., 217(2), 545-556.

Holmes, P.J. (1978), "Pipes supported at both ends cannot flutter", J. Appl. Mech., 45(3), 619-622.

Huang, J.L., Su, R.K.L., Li, W.H. and Chen, S.H. (2011), "Stability and bifurcation of an axially moving beam tuned to three-to-one internal resonances", J. Sound Vib., 330(3), 471-485.

Kazemirad, S., Ghayesh, M.H. and Amabili, M. (2012), "Thermo-mechanical nonlinear dynamics of a buckled axially moving beam", Arch. Appl. Mech., in press.

Marynowski, K. and Kapitaniak, T. (2002), "Kelvin-Voigt versus Bürgers internal damping in modeling of axially moving viscoelastic web", Int. J. Nonlinear Mech., 37(7), 1147-1161.

Marynowski, K. and Kapitaniak, T. (2007), "Zener internal damping in modelling of axially moving viscoelastic beam with time-dependent tension", Int. J. Nonlinear Mech., 42(1), 118-131.

Naguleswaran, S. and Williams, C.J.H. (1968), "Lateral vibration of band-saw blades, pulley belts and the like", Int. J. Mech. Sci., 10(4), 239-250.

Nguyen, Q.C. and Hong, K.S. (2011), "Stabilization of an axially moving web via regulation of axial velocity", J. Sound Vib., 330(20), 4676-4688.

Öz, H.R., Pakdemirli, M. and Boyaci, H. (2001), "Non-linear vibrations and stability of an axially moving beam with time-dependent velocity", Int. J. Nonlinear Mech., 36(1), 107-115.

Pakdemirli, M. and BoyacI, H. (2003), "Non-linear vibrations of a simple-simple beam with a non-ideal support in between", J. Sound Vib., 268(2), 331-341.

Pakdemirli, M. and Özkaya, E. (1998), "Approximate boundary layer solution of a moving beam problem", Math. Comput. Appl., 3, 93-100.

Pakdemirli, M. and Ulsoy, A.G. (1997), "Stability analysis of an axially accelerating string", J. Sound Vib., 203(5), 815-832.

Pakdemirli, M., Ulsoy, A.G. and Ceranoglu, A. (1994), "Transverse vibration of an axially accelerating string", $J$. Sound Vib., 169(2), 179-196.

Pellicano, F. and Vestroni, F. (2002), "Complex dynamics of high-speed axially moving systems", J. Sound Vib., 258(1), 31-44.

Shih, L.Y. (1971), “Three-dimensional non-linear vibration of a traveling string”, Int. J. Nonlinear. Mech., 6(4), 427-434.

Simpson, A. (1973), "Transverse modes and frequencies of beams translating between fixed end supports", $J$. Mech. Eng. Sci, 15, 159-164.

Stylianou, M. and Tabarrok, B. (1994), "Finite element analysis of an axially moving beam, Part I: time integration", J. Sound Vib., 178(4), 433-453.

Suweken, G. and Van Horssen, W.T. (2003), "On the weakly nonlinear, transversal vibrations of a conveyor belt with a low and time-varying velocity”, Nonlinear Dynam., 31(2), 197-223. 
Tang, Y.Q., Chen, L.Q. and Yang, X.D. (2009), "Parametric resonance of axially moving Timoshenko beams with time-dependent speed", Nonlinear Dynam., 58(4), 715-724.

Thurman, A.L. and Mote, C.D.J. (1969), "Free, periodic, nonlinear oscillation of an axially moving strip", $J$. Appl. Mech. - ASME, 36(1), 83-91.

Wickert, J.A. and Mote, C.D., Jr. (1988), "Current research on the vibration and stability of moving materials", Shock Vib., 20(5), 3-13.

Yang, X.D., Zhang, W., Chen, L.Q. and Yao, M.H. (2012), "Dynamical analysis of axially moving plate by finite difference method", Nonlinear Dynam., 67(2), 997-1006. 